\title{
A teenager presenting with pain and popliteal mass
}

\author{
Eduardo Baptista $^{1} \cdot$ Rafael Kubo $^{1}$ - Durval C. B. Santos ${ }^{1}$ - Atul K. Taneja ${ }^{1,2}$
}

Published online: 9 March 2017

(C) ISS 2017

Keywords Osteochondroma · Exostosis · Pseudoaneurysm · Popliteal mass $\cdot$ MRI $\cdot$ Doppler ultrasound

\section{Pseudoaneurysm of the popliteal artery secondary to a distal femoral osteochondroma}

Osteochondromas are a relatively common imaging finding, comprising 20-50\% of benign bone tumors [1]. They are often found in metaphyseal regions of long bones, protruding away from the epiphysis [2]. Their appearance is typical on conventional radiographs (Fig. 1), with the main feature being the continuity of the lesion with underlying native cortex and cancellous bone, as well as the presence of a cartilage cap [3], which is best evaluated on MRI (Fig. 2) [2]. The majority of these masses are asymptomatic [2]. The diagnosis is commonly incidental or secondary to related complications, such as fractures, vascular or neurologic compromise, adventitious bursa formation, or malignant transformation [1].

Vascular injuries secondary to osteochondromas were first reported by Paul et al. in 1953, who described a complex mass intimately related to the underlying artery [4]. These abnormalities are common in adolescent and young-adult males, possibly related to vigorous physical activity. Furthermore, they may

The case presentation can be found at doi: 10.1007/s00256-017-2600-2

Atul K. Taneja

taneja@einstein.br

1 Musculoskeletal Radiology Division, Imaging Department, Hospital Israelita Albert Einstein, São Paulo, Brazil

2 Departamento de Imagem, Hospital Israelita Albert Einstein, Av. Albert Einstein, 627, Morumbi, São Paulo, SP, Brazil CEP 05652-900 develop in the setting of solitary or multiple bone exostoses, being associated with trauma in one-third of cases [5].

Vascular injury may occur when there is an abnormal proximity between a bone exostosis and the adventitial vessel wall, leading to friction and tearing. The popliteal artery is especially susceptible to injury as compared to the popliteal vein, which can be partially credited to its anatomic features $[5,6]$. The artery is fixed proximally at the aponeurotic hiatus of the adductor magnus, while it is distally tethered to its trifurcation. These circumstances provide little mobility and increased vulnerability to erosive forces during vascular pulsation and knee motion. Damage to the vessel wall may lead to pseudoaneurysm formation, although other abnormalities such as vascular compression and thrombosis also have been reported [5].

Ultrasound Doppler, CT, and MR angiography are useful in investigating vascular-related injuries; however, an inaccurate history or unrecognized bone exostosis may lead to incorrect clinical assessment and suggesting a soft tissue tumor. In this setting, conventional MRI is often requested and although not the ideal tool, may provide substantial information. The radiologist's experience is critical in these situations and they must be able to recognize the features of a pseudoaneurysm.

The hallmark of pseudoaneurysm by MRI is that the signal intensity of soft tissue varies according to the temporal metabolization of the blood (Fig. 2). Areas showing T1 shortening of methemoglobin (high signal intensity on T1weighted images) and T2 shortening of hemosiderin (low signal intensity on T2-weighted images) are characteristic, in addition to blooming artifact in gradient-echo sequences and fluid levels [1]. Imaging after intravenous administration of gadolinium-based contrast material may reveal enhancement in the cartilage cap, the adventitial bursal wall and the pseudoaneurysm itself [7]. Doppler ultrasound classically exhibits the swirling motion known as the "yin-yang sign" and a "to-and-fro" waveform pattern at duplex scan [7]. 
The patient underwent surgery for hematoma drainage; closure of the arterial defect and the osteochondroma was excised at its base. Histological examination confirmed the diagnosis, without evidence of malignancy.

\section{Compliance with ethical standards}

Disclosure No conflicts of interests.

\section{References}

1. Murphey MD, Choi JJ, Kransdorf MJ, Flemming DJ, Gannon FH. Imaging of osteochondroma: variants and complications with radiologic-pathologic correlation. Radiographics. 2000;20(5):1407-34.
2. Lee JK, Yao L, Wirth CR. MR imaging of solitary osteochondromas: report of eight cases. AJR. 1987;149:557-60.

3. Karasick D, Schweitzer ME, Eschelman DJ. Symptomatic osteochondromas: imaging features. AJR Am J Roentgenol. 1997;168(6):1507-12.

4. Paul M. Aneurysm of the popliteal artery from perforation by a cancellous exostosis of the femur. J Bone Joint Surg (Br). 1953;35$\mathrm{B}(2): 270-1$.

5. Taneda Y, Nakamura K, Yano M, et al. Popliteal artery pseudoaneurysm caused by osteochondroma. Ann Vasc Surg. 2004;18(1):121-3.

6. Perez-Burkhardt JL, Gómez Castilla JC. Postraumatic popliteal pseudoaneurysm from femoral osteochondroma: case report and review of the literature. J Vasc Surg. 2003;37(3):669-71.

7. Mahmoud MZ, Mohammed A-S, Abdulwahab A, et al. "To-andfro" waveform in the diagnosis of arterial pseudoaneurysms. World J Radiol. 2015;7(5):89-99. 